\title{
The effect of orbital implantation on peripheral blood melatonin and sex hormone levels in child patients with congenital eyeball dysplasia
}

\author{
JUNZE MA ${ }^{1}$, TAO LIU ${ }^{2}$ and JIANQIANG QU ${ }^{3}$ \\ ${ }^{1}$ Department of Ophthalmology, The Second Affiliated Hospital of Xi'an Jiaotong University, Xi'an, \\ Shaanxi 710004; ${ }^{2}$ Department of Ophthalmology, The Central Hospital of Baoji City, Baoji, Shaanxi 721008; \\ ${ }^{3}$ Department of Neonatal Medicine, The Central Hospital of Shangluo City, Shangluo, Shaanxi 726000, P.R. China
}

Received March 31, 2017; Accepted June 27, 2017

DOI: 10.3892/etm.2017.4719

\begin{abstract}
The aim of the study was to examine the effect of orbital implantation on peripheral blood melatonin and sex hormone levels in pediatric patients with congenital eyeball dysplasia. A total of 28 cases of pediatric patients with congenital eyeball dysplasia diagnosed in the Second Affiliated Hospital of Xi'an Jiaotong University from June 2014 to December 2014 were selected for the study. The patients included those that received orbital implantation, and the melatonin levels in the peripheral blood in patients before and after operation was observed. In addition, the sex hormone levels and $\mathrm{T}$ lymphocytes, plasma reactive oxygen species (ROS) and VEGF levels, urine 8-OHdG and 8 -isoPGF $2 \alpha$ levels in patients before and after treatment were detected, followed by statistical analysis. As a result, after 3 months of orbital implantation, the sex hormone levels in peripheral blood in child patients fluctuated significantly, and differences were not statistically significant $(\mathrm{P}>0.05)$. The peripheral blood $\mathrm{T}$ lymphocytes and ROS levels were significantly lower than those before treatment, and the differences were statistically significant $(\mathrm{P}<0.05)$. The correlation analysis revealed that the peripheral blood melatonin levels were negatively related to ROS levels; the correlation coefficient was $\mathrm{rs}=-0.481$ and $\mathrm{P}<0.05$. In conclusion, orbital implantation does not have significant impact on sex hormone levels in child patients with congenital eyeball dysplasia. The hydroxyapatite orbital implantation can achieve more satisfactory curative effects, and there are fewer postoperative complications. It does not affect the appearance of the eye, and therefore, it is suitable for patients with congenital eyeball dysplasia.
\end{abstract}

Correspondence to: Dr Jianqiang Qu, Department of Neonatal Medicine, The Central Hospital of Shangluo City, 148 Water Street, Shangzhou, Shangluo, Shaanxi 726000, P.R. China

E-mail: qigsgw@163.com

Key words: melatonin, orbital implantation, reactive oxygen radical

\section{Introduction}

Congenital eyeball dysplasia is a type of congenital eyeball development disorder. Pediatric patients often have trouble expressing any issues and thus, this disease is usually diagnosed and treated in the physical examination for enrollment, except for patients with severe development disorder $(1,2)$. Currently, congenital eyeball dysplasia is mainly treated through orbital implantation, which can reduce the effect of enucleation on the appearance and facial development of pediatric patients $(3,4)$.

Melatonin is a type of amine hormone mainly produced by the pineal body of mammals and humans. It can adjust the reproductive activity of photoperiodic animals (5). Previous findings have shown that the melatonin receptor is present in ovaries, suggesting that melatonin is of great significance to the reproductive system $(6,7)$. The secretion of melatonin is correlated with light. Thus, the relationship between melatonin secretion levels and sex hormone secretion is unclear in child patients with congenital eyeball dysplasia.

Through the detection of peripheral blood T lymphocytes and ROS levels before and after melatonin treatment in child patients with congenital eyeball dysplasia, further shows the role and significance of melatonin in congenital eyeball dysplasia, thus providing theoretical support for the clinical treatment of melatonin.

\section{Materials and methods}

Sample selection. A total of 28 cases of pediatric patients with congenital eyeball dysplasia diagnosed in the Optometry Department in the Second Affiliated Hospital of Xi'an Jiaotong University from June 2014 to December 2014 were selected.

Inclusion criteria. The inclusion criteria for the study were: i) Patients aged $<18$, and ii) clinical diagnosis of congenital eyeball dysplasia via the laboratory examination.

Exclusion criteria. The exclusion criteria for the study were: i) Patients with congenital cataracts; ii) patients with complicated malignant tumors of other systems; iii) patients without 
a definite diagnosis; iv) patients with cognitive impairment or mental disease; v) patients whose sample could not be obtained due to some reasons; vi) patients or their families who failed to cooperate; vii) patients who dropped out of the study due to some reasons; and viii) patients in poor condition, and not suitable for the diagnosis and treatment.

Blood collection. Fasting elbow venous blood $(3 \mathrm{ml})$ was taken and injected into the common plastic tube, and $1.8 \mathrm{ml}$ of blood was injected into an anticoagulant tube containing $0.2 \mathrm{ml}$ of $3.8 \%$ sodium citrate. The specimen was centrifuged at 2,650 $\mathrm{x} \mathrm{g}$ for $10 \mathrm{~min}$ within $1 \mathrm{~h}$ after collection. The serum or plasma was extracted and placed into a $0.5 \mathrm{ml} \mathrm{EP}$ tube, respectively, stored under $-30^{\circ} \mathrm{C}$ and tested within 1 month.

Experiment reagents. DCFH-DA powder (Sigma-Aldrich, St. Louis, MO, USA), double-distilled water, anti-phosphorylation TGF- $\beta 1$ (p-TGF- $\beta 1)$ (1:1,000; Cell Signaling Technology Inc., Danvers, MA, USA), $\beta$-actin antibody $(1: 5,000$; Invitrogen, Carlsbad, CA, USA), $0.9 \%$ sterile saline solution (Otsuka Pharmaceutical, Co., Ltd., Tokyo, Japan) and TRIzol (Invitrogen) were obtained commercially.

Experimental apparatus. Centrifugal machine (Eppendorf AG, Hamburg, Germany), micropipettor (Eppendorf), ice machine (Qingdao Haier Co., Ltd, Qingdao, China), western blot electrophoresis apparatus (Bio-Rad Laboratories, Inc., Hercules, CA, USA), $-80^{\circ} \mathrm{C}$ refrigerator (Thermo Fisher Scientific, Waltham, MA, USA), $10 \mathrm{ml}$ syringe, $5 \mathrm{ml}$ syringe (Tianjin Hanaco Medical Co., Ltd., Tianjin, China), special surgical instruments for experimental animals (Beijing Medical Device Factory, Beijing, China), NanoDrop2000 photometer (Thermo Fisher Scientific), EP tube (Eppendorf), water bath kettle (Beijing Medical Device Factory), pathologic microtome (Leica Microsystems GmbH, Wetzlar, Germany), and flow cytometer (PARTEC CyFlow ${ }^{\circledR}$; Partec Münster, Germany) were obtained commercially.

Surgical method. Surgical preparation was carried out as previously described (8). The Hydroxyapatite orbital implantation with a micro-pore diameter of 200-500 $\mu$ was used. There were many diameter specifications, such as 22, 20, 18 and $16 \mathrm{~mm}$, as well as the sterile packaging. Before implantation, the orbit implant was soaked in $0.2 \%$ gentamicin saline solution for $15 \mathrm{~min}$. Allogeneic sclera was prepared as follows: an allogeneic sclera shell stored in anhydrous alcohol was soaked in $0.2 \%$ gentamicin saline solution for $1-2 \mathrm{~h}$, and the pigment and muscle tissue attached inside and outside the sclera were removed; after which the sclera shell was washed using normal saline in reserve. Autologous or allogeneic sclera-wrapped orbit implantation was conducted as the conventional method. The orbit implant was wrapped using treated autologous or allogeneic sclera with an optic nerve forward, and it was implanted inside the eye muscle cone. The fascia bulbi and bulbar conjunctiva were sutured in different layers. Non-sclerawrapped orbit implant with the suture and the orbit implant with the suture was implanted inside the eye muscle cone. The four preplaced sutures of orbit implant were directly connected with four rectus preplaced sutures, and then the fascia bulbi and bulbar conjunctiva were sutured in different layers. Those with narrow and shallow conjunctival sac received both orbital implantation and conjunctival sac plasty.

Detection of human plasma ROS and VEGF levels using ELISA. After the centrifugation and supernatant extraction, ROS and VEGF levels were detected using human serum ROS kit and VEGF kit according to specification.

Extraction method of T lymphocytes. T lymphocytes were extracted as follows: i) An appropriate amount of lymphocyte separating medium was added into the short and medium tube; ii) the heparin anti-coagulation vein blood was mixed uniformly with the same amount of Hank's fluid or RPMI-1640, and was added along the wall with a dropper slowly into the stratified fluid surface. The clear interface was kept and the fluid was centrifuged horizontally at $1,750 \mathrm{x} g$ for $20 \mathrm{~min}$; iii) the liquid in the tube was divided into three layers after the centrifugation: the upper layer contained the plasma and Hank's fluid, the lower layer mainly contained red blood cells and granulocytes, and the middle layer contained the lymphocyte separation medium. Between the upper and middle layer was the white cloud layer narrow belt with mainly mononuclear cells, and the mononuclear cells included the lymphocytes and monocytes. In addition, this also contained platelets; iv) the capillary was inserted into the cloud layer to draw mononuclear cells. The mononuclear cells were placed into another short and medium tube, and Hank's fluid or RPMI-1640 with >5 times of volume was added and the solution was centrifuged at 1,200 $\mathrm{x}$ g for $10 \mathrm{~min}$, and the cells were washed twice and v) after the last centrifugation, the supernatant was discarded, and RPMI-1640 containing $10 \%$ calf serum was added and the cells were re-suspended. One drop of cell suspension and one drop of $0.2 \%$ trypan blue were mixed and placed on a blood counting chamber, and the total cell number in the four big grids were counted.

Urine oxidative stress detection. The morning urine of child patients was collected after 6 weeks, and stored in a $-80^{\circ} \mathrm{C}$ refrigerator immediately for later testing. The ELISA method was applied to detect the 8 -OHdG and 8-isoPGF2 $\alpha$ levels (kit; Cayman Chemical Company, Ann Arbor, MI, USA). The NT level in urine was detected using the chemiluminescence method (kit; Millipore Corp., Bedford, MA, USA).

Statistical analysis. The SPSS19.0 statistical analysis software (Chicago, IL, USA)was used in this study for data processing and analysis. Continuous variables were presented as the mean \pm standard deviation. Continuous variables were described using median and interquartile range when they were in skewed distribution. Classified variables were presented as the absolute percentage. The Student's t-test was used for the continuous variables and $\chi^{2}$ test was used for classified variables. $\mathrm{P}<0.05$ suggested that the difference was statistically significant.

\section{Results}

Comparison of sex hormone levels in child patients before and after the treatment. After 6 months of treatment, the peripheral blood sex hormone levels in pediatric patients 
Table I. Comparison of sex hormone levels in child patients before and after the treatment.

\begin{tabular}{|c|c|c|c|c|c|c|c|}
\hline Sex & Item & $\begin{array}{l}\text { Before } \\
\text { treatment }\end{array}$ & $\begin{array}{l}1 \text { month } \\
\text { after treatment }\end{array}$ & $\begin{array}{l}3 \text { months } \\
\text { after treatment }\end{array}$ & $\begin{array}{l}6 \text { months } \\
\text { after treatment }\end{array}$ & F-value & $\mathrm{P}$ value \\
\hline \multirow[t]{3}{*}{ Female } & $\begin{array}{l}\text { Follicle-stimulating } \\
\text { hormone }(\mathrm{pmol} / \mathrm{l})\end{array}$ & $15.3 \pm 3.6$ & $13.3 \pm 1.6$ & $12.4 \pm 1.6$ & $15.49 \pm 2.3$ & 0.39 & 0.65 \\
\hline & $\begin{array}{l}\text { Luteinizing hormone } \\
(\mathrm{pmol} / \mathrm{l})\end{array}$ & $10.4 \pm 9.1$ & $12.4 \pm 2.3$ & $15.3 \pm 3.4$ & $10.4 \pm 2.1$ & 0.29 & 0.71 \\
\hline & $\begin{array}{r}\text { Estradiol } \\
(\mathrm{pmol} / \mathrm{l})\end{array}$ & $62.7 \pm 33.4$ & $69.3 \pm 12.5$ & $68.4 \pm 23.1$ & $62.7 \pm 13.4$ & 0.44 & 0.38 \\
\hline Male & $\begin{array}{l}\text { Testosterone } \\
\text { (T) }(\mathrm{mg} / \mathrm{l})\end{array}$ & $2.3 \pm 1.2$ & $2.4 \pm 2.1$ & $2.3 \pm 1.2$ & $3.2 \pm 1.2$ & 0.44 & 0.52 \\
\hline
\end{tabular}

Table II. Comparison of plasma ROS and VEGF levels in child patients before and after treatment.

\begin{tabular}{lccccc}
\hline Inspection item & $\begin{array}{c}\text { Before } \\
\text { treatment }\end{array}$ & $\begin{array}{c}1 \text { month } \\
\text { after treatment }\end{array}$ & $\begin{array}{c}3 \text { months } \\
\text { after treatment }\end{array}$ & $\begin{array}{c}6 \text { months } \\
\text { after treatment }\end{array}$ & $\begin{array}{c}\text { F-value } \\
\text { P-value }\end{array}$ \\
\hline ROS $(\mathrm{ng} / \mathrm{ml})$ & $203.4 \pm 21.2$ & $167.4 \pm 12.1$ & $123.2 \pm 13.5$ & $58.5 \pm 22.7$ & 44.37 \\
MLT $(\mathrm{ng} / \mathrm{ml})$ & $10.7 \pm 9.4$ & $12.3 \pm 2.6$ & $22.5 \pm 10.7$ & $48.5 \pm 12.6$ & 24.3 \\
\hline
\end{tabular}

ROS, reactive oxygen species.

Table III. Urine oxidative stress detection result.

\begin{tabular}{lcccc}
\hline & \multicolumn{2}{c}{8 -OHdG } & \multicolumn{2}{c}{8 -isoPGF2 $\alpha$} \\
\cline { 2 - 4 } Item & Before treatment & After treatment & Before treatment & After treatment \\
\hline Value & $15.7 \pm 3.4$ & $11.5 \pm 1.6^{\mathrm{a}}$ & $14.3 \pm 1.4$ & $11.5 \pm 2.5^{\mathrm{b}}$ \\
T-value & 0.11 & 21.7 & 36.5 & 34.2 \\
P-value & 0.83 & 0.01 & 0.008 & 0.007 \\
\hline
\end{tabular}

${ }^{\mathrm{a}}$ Compared with that before treatment, $\mathrm{T}=12.5, \mathrm{P}<0.05 .{ }^{\mathrm{b}}$ Compared with that before treatment, $\mathrm{T}=21.3, \mathrm{P}<0.05$

after 6 months of treatment did not fluctuate significantly when compared to before the treatment; differences were not statistically significant $(\mathrm{P}>0.05)$. This result suggested that the orbital implantation did not affect the normal gonad function in pediatric patients (Table I).

Comparison of plasma melatonin and ROS level in child patients before and after the treatment. The comparison of plasma ROS levels in pediatric patients before and after treatment showed that after 6 months of operation, the plasma ROS levels were significantly decreased and MLT levels were significantly increased. Differences were statistically significant compared to the preoperative levels $(\mathrm{P}<0.05$, Table II).

Urine oxidative stress detection result. The urine oxidative stress levels of $8-\mathrm{OHdG}$ and 8 -isoPGF2 $\alpha$ in pediatric patients after treatment were significantly decreased as compared to before and after treatment. The difference was statistically significant when compared to the levels before treatment $(\mathrm{P}<0.05)$ (Table III).

Correlation analysis of serum melatonin level and ROS level. We found, through the correlation analysis of serum melatonin levels and ROS levels in pediatric patients, that the serum melatonin levels were negatively correlated with ROS levels. Differences were statistically significant $(\mathrm{P}<0.05)$, and the correlation coefficient was $\mathrm{rs}=0.481$ (Tables IV and V).

\section{Discussion}

Pediatric patients with congenital eyeball dysplasia suffer from sunken orbital socket after enucleation or evisceration of eyeballs, and many complications occur if there is no filling, such as sunken upper eyelid, lower eyelid laxity, shallow 
Table IV. Correlation analysis of ROS level and clinical test index.

\begin{tabular}{llllllll}
\hline Index & Sex & Age & FSH & LH & Estradiol & ROS & BMI $\left(\mathrm{kg} / \mathrm{m}^{2}\right)$ \\
\hline $\begin{array}{l}\text { Melatonin } \\
\text { r }\end{array}$ & 0.021 & 0.361 & 0.174 & 0.161 & 0.074 & -0.481 & 0.141 \\
P-value & 0.33 & 0.58 & 0.71 & 0.62 & 0.32 & $<0.05$ & $<0.05$ \\
\hline
\end{tabular}

ROS, reactive oxygen species.

Table V. Multiple linear regression analysis.

\begin{tabular}{|c|c|c|c|c|c|c|c|}
\hline \multirow[b]{2}{*}{ Variable } & \multirow[b]{2}{*}{$\beta$} & \multirow[b]{2}{*}{ SE } & \multirow[b]{2}{*}{$\beta^{\prime}$} & \multirow[b]{2}{*}{ t-test } & \multirow[b]{2}{*}{ P-value } & \multicolumn{2}{|c|}{$(95 \% \mathrm{CI})$} \\
\hline & & & & & & $\begin{array}{l}\text { Upper } \\
\text { limit }\end{array}$ & $\begin{array}{c}\text { Lower } \\
\text { limit }\end{array}$ \\
\hline BMI & 0.531 & 0.14 & 0.764 & 0.412 & $>0.05$ & 0.26 & 0.81 \\
\hline ROS & 0.768 & 0.08 & 0.871 & 0.981 & $<0.05$ & 0.61 & 0.92 \\
\hline
\end{tabular}

ROS, reactive oxygen species.

conjunctival sac or abnormal shape. In particular, it affects the orbital and ipsilateral facial development of children $(1,2)$. The causes of enucleation and ocular evisceration are complicated, and therefore, the orbital implant and implantation are also different. The HA orbital implant is characterized by high biocompatibility, no poison and no rejection. Moreover, the porous feature is conducive to vascularization, thus it is an ideal orbital implant material $(1,3)$. This surgical method and procedure continue to be explored and improved clinically. However, there is also controversy surrounding the use of autologous or allogeneic material packaging in orbital implantation $(4,5)$. It is generally believed that the advantages of sclera packaging include non-toxicity and no irritation with low antigenicity. Since rejection reaction seldom occurs after processing sclera packaging can be used as a shield to reduce the friction between orbit implant and fascia bulbi, which is conducive to wound healing. However, this type of surgery causes larger operative wounds and the operation is complicated. In addition, when autologous sclera cannot be used, the application is restricted by the material source of allogeneic sclera. The advantages of non-sclera packaging include that the surgical procedure is relatively simple, the orbit implant directly contacts orbital tissues, and therefore, it is advantageous to vascular ingrowth. Allogeneic sclera is not needed, especially when autologous sclera cannot be used and the occurrence of postoperative rejection reaction is also reduced. It is also one of the main treatment methods of congenital eyeball dysplasia.

Melatonin is a type of hormone secreted by the pineal gland at night. Its secretion is periodic, and maximum secretion occurs at midnight. Studies have shown that melatonin peak levels are related to various disease factors, such as age (9). Melatonin is important in sleep-wake regulation. In addition, melatonin can also regulate growth and development, and seasonal adaptability of the body in adolescence jointly with growth hormone (10). The present study has confirmed that the secretion levels of melatonin in the human body are closely related to the memory ability, body posture and balance control (11). Melatonin can directly affect the hippocampal neurons in order to adjust the formation and persistence of memory (12). In addition, melatonin has an analgesic, antidepression and anti-anxiety effect, and it can regulate the body's autonomic activities (13). Melatonin plays a biological effect in nerve protection, anti-inflammatory, abirritation, blood pressure decrease, retinal vessel, seasonal reproduction, ovarian physiology, osteoblast differentiation, anti-tumor and antioxidant effects $(14,15)$. Therefore, after orbital implantation, whether the endocrine function is affected in the development process of pediatric patients has been one of the hot topics in the current study.

In this study, we found that after orbital implantation, the endocrine hormone levels in pediatric patients with congenital eyeball dysplasia were not significantly affected, but the peripheral blood T-lymphocyte ROS levels were significantly decreased $(\mathrm{P}<0.05)$. The results suggest that the orbital implantation does not affect the endocrine function of pediatric patients and orbital implantation has a certain effect on improving the ROS levels in the body because it improves the rhythmic change of daily secretion of melatonin in pediatric patients.

ROS mainly refers to a series of reactive oxygen species (ROS) in the human body produced during the process of aerobic metabolism, including $\mathrm{O}_{2^{-}}, \mathrm{H}_{2} \mathrm{O}_{2}, \mathrm{HO}_{2}{ }^{\circ}, \mathrm{OH}$ (16). Medium and high-concentration ROS induces cell apoptosis and even necrosis by cellular oxidative stress reactions. The development of free-radical biology research has shown that ROS can regulate apoptosis and proliferation of some tumor cells in both directions. In addition, we found that the plasma 
ROS and VEGF levels in pediatric patients receiving melatonin treatment in the observation group usually decreased significantly with the extension of treatment time. However, such a change did not exist in pediatric patients of the control group. Melatonin is a type of non-enzyme antioxidant substance with stable structure, and auto-oxidation does not occur easily. Therefore, it can remove -OH and different types of ROS, exerting an anti-oxidation effect (17-20).

Melatonin reduces the production of ROS. Through the use of in vivo and in vitro experiments, it has been identified that melatonin can inhibit hypoxia-inducible factor HIF-1, HIF-1-induced gene, and vascular endothelial growth factor. This occurs through the HIF-1-VEGF pathway; whereby melatonin can reduce the hypoxia-induced angiogenesis. Mehrzadi et al (21) found that the ability of mannitol (MT) to remove -OH was 5 times that of glutathione (GSH), and 8 times that of MT. MT also has an indirect antioxidant effect that can promote the expression of antioxidant defensive enzyme gene via nuclear receptor-mediated mechanism, and increases the activity of antioxidant enzymes.

However, at the same time, this study has certain limitations. Anti-ROS is a complex pathological and physiological process in the body and thus, such a process usually requires a variety of inflammatory factors and signaling pathways. Simple detection in the change of VEGF cannot fundamentally explain the relationship between such factors and pathways. We thought that the improvement of melatonin on ROS levels can be further proved in animal experiments.

Based on the results of this study and the review and analysis of previous studies, we believe that orbital implantation can improve the secretion rhythm of melatonin and reduce ROS production in the body of pediatric patients with congenital eyeball dysplasia, and thus, it does not affect normal sex hormone secretion in patients. Therefore, orbital implantation is of clinical significance.

\section{Acknowledgements}

This study was supported by the Natural Science Foundation of China (no. 81601222).

\section{References}

1. Cogan DG: A type of congenital ocular motor apraxia presenting jerky head movements. Trans Am Acad Ophthalmol Otolaryngol 56: 853-862, 1952.

2. Harris CM, Shawkat F, Russell-Eggitt I, Wilson J and Taylor D: Intermittent horizontal saccade failure ('ocular motor apraxia') in children. Br J Ophthalmol 80: 151-158, 1996.

3. Zee DS, Yee RD and Singer HS: Congenital ocular motor apraxia. Brain 100: 581-599, 1977.

4. Rappaport L, Urion D, Strand K and Fulton AB: Concurrence of congenital ocular motor apraxia and other motor problems: An expanded syndrome. Dev Med Child Neurol 29: 85-90, 1987.

5. Steinlin M, Thun-Hohenstein L and Boltshauser E: Congenital oculomotor apraxia. Presentation - developmental problems - differential diagnosis. Klin Monbl Augenheilkd 200: 623-625, 1992 (In German).
6. Inkster CF, Stephen GJNg and Leatherbarrow B: Primary banked scleral patch graft in the prevention of exposure of hydroxyapatite orbital implants. Ophthalmology 109: 389-392, 2002.

7. Remulla HD, Rubin PA, Shore JW, Sutula FC, Townsend DJ, Woog JJ and Jahrling KV: Complication of porous spherical orbital implants. Ophthalmol 102: 586-593, 1995.

8. De Potter P, Shields CL and Shields JA: Use of the hydroxyapatite ocular implant in the pediatric population. Arch Ophthalmol 112: 208-212, 1994.

9. Pelletier CR, Jordan DR and Gilberg SM: Use of temporalis fascia for exposed hydroxyapatite orbital implants. Ophthal Plast Reconstr Surg 14: 198-203, 1998.

10. Ambati J, Atkinson JP and Gelfand BD: Immunology of age-related macular degeneration. Nat Rev Immunol 13: 438-451, 2013.

11. Bowes Rickman C, Farsiu S, Toth CA and Klingeborn M: Dry age-related macular degeneration: Mechanisms, therapeutic targets, and imaging. Invest Ophthalmol Vis Sci 54: ORSF68-80, 2013.

12. Klein R, Myers CE, Cruickshanks KJ, Gangnon RE, Danforth LG, Sivakumaran TA, Iyengar SK, Tsai MY and Klein BE: Markers of inflammation, oxidative stress, and endothelial dysfunction and the 20-year cumulative incidence of early age-related macular degeneration: The beaver dam eye study. JAMA Ophthalmol 132: 446-455, 2014.

13. Kwak N, Okamoto N, Wood JM and Campochiaro PA: VEGF is major stimulator in model of choroidal neovascularization. Invest Ophthalmol Vis Sci 41: 3158-3164, 2000.

14. Heckenlively JR, Hawes NL, Friedlander M, Nusinowitz S, Hurd R, Davisson M and Chang B: Mouse model of subretinal neovascularization with choroidal anastomosis. Retina 23: 518-522, 2003.

15. Lopez PF, Sippy BD, Lambert HM, Trach AB and Hinton DR: Transdifferentiated retinal pigment epithelial cells are immunoreactive for vascular endothelial growth factor in surgically excised age-related macular degeneration-related choroidal neovascular membranes. Invest Ophthalmol Vis Sci 37: 855-868, 1996.

16. Benedict C, Thom B, Friedman DN, Diotallevi D, Pottenger EM, Raghunathan NJ and Kelvin JF: Young adult female cancer survivors' unmet information needs and reproductive concerns contribute to decisional conflict regarding posttreatment fertility preservation. Cancer 122: 2101-2109, 2016.

17. Check JH, Wilson C, DiAntonio G and DiAntonio A: In vitro fertilization (IVF) outcome in women in overt menopause attempting to induce follicular maturation by follicle stimulating hormone (FSH) receptor down-regulation. Clin Exp Obstet Gynecol 43: 181-183, 2016.

18. Sundaresan NR, Marcus Leo MD, Subramani J, Anish D, Sudhagar M, Ahmed KA, Saxena M, Tyagi JS, Sastry KV and Saxena VK: Expression analysis of melatonin receptor subtypes in the ovary of domestic chicken. Vet Res Commun 33: 49-56, 2009.

19. Liu T, Li Q, Wang S, Chen C and Zheng J: Transplantation of ovarian granulosa like cells derived from human induced pluripotent stem cells for the treatment of murine premature ovarian failure. Mol Med Rep 13: 5053-5058, 2016.

20. Kleszczyński K, Zillikens D and Fischer TW: Melatonin enhances mitochondrial ATP synthesis, reduces ROS formation and mediates translocation of the nuclear erythroid 2-related factor 2 resulting in activation of phase-2 antioxidant enzymes ( $\gamma$-GCS, HO-1, NQO1) in UVR-treated normal human epidermal keratinocytes (NHEK). J Pineal Res 61: 187-197, 2016.

21. Mehrzadi S, Kamrava SK, Dormanesh B, Motevalian M, Hosseinzadeh A, Hosseini Tabatabaei SM and Ghaznavi H: Melatonin synergistically enhances protective effect of atorvastatin against gentamicin-induced nephrotoxicity in rat kidney. Can J Physiol Pharmacol 94: 265-271, 2016. 